\title{
Simulating marine propellers with vortex particle method
}

, Moustafa Abdel-Maksoud, and

Citation: Phys. Fluids 29, 017103 (2017); doi: 10.1063/1.4974522

View online: http://dx.doi.org/10.1063/1.4974522

View Table of Contents: http://aip.scitation.org/toc/phf/29/1

Published by the American Institute of Physics

\section{Articles you may be interested in}

Aerodynamics of two-dimensional flapping wings in tandem configuration

Phys. Fluids 28, 121901121901 (2016); 10.1063/1.4971859

Experimental study on the multimodal dynamics of the turbulent horseshoe vortex system around a circular cylinder

Phys. Fluids 29, 015106015106 (2017); 10.1063/1.4974523

Swimming of a linear chain with a cargo in an incompressible viscous fluid with inertia

Phys. Fluids 29, 011901011901 (2017); 10.1063/1.4973711

A paradigm for modeling and computation of gas dynamics

Phys. Fluids 29, 026101026101 (2017); 10.1063/1.4974873

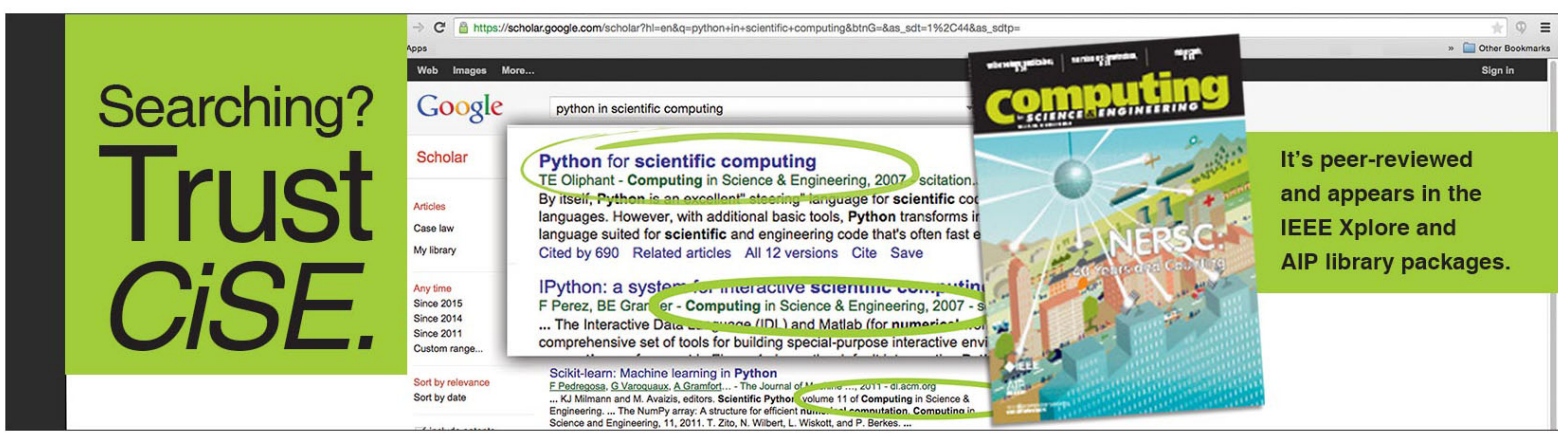




\title{
Simulating marine propellers with vortex particle method
}

\author{
Youjiang Wang (王有江), ${ }^{1, a)}$ Moustafa Abdel-Maksoud, ${ }^{2}$ and Baowei Song (宋保维) ${ }^{1}$ \\ ${ }^{1}$ School of Marine Science and Technology, Northwestern Polytechnical University, Xi'an 710072, China \\ ${ }^{2}$ Institute for Fluid Dynamics and Ship Theory, Hamburg University of Technology, Am Schwarzenberg-Campus \\ 4, 21073 Hamburg, Germany
}

(Received 12 August 2016; accepted 6 January 2017; published online 31 January 2017)

\begin{abstract}
The vortex particle method is applied to compute the open water characteristics of marine propellers. It is based on the large-eddy simulation technique, and the Smagorinsky-Lilly sub-grid scale model is implemented for the eddy viscosity. The vortex particle method is combined with the boundary element method, in the sense that the body is modelled with boundary elements and the slipstream is modelled with vortex particles. Rotational periodic boundaries are adopted, which leads to a cylindrical sector domain for the slipstream. The particle redistribution scheme and the fast multipole method are modified to consider the rotational periodic boundaries. Open water characteristics of three propellers with different skew angles are calculated with the proposed method. The results are compared with the ones obtained with boundary element method and experiments. It is found that the proposed method predicts the open water characteristics more accurately than the boundary element method, especially for high loading condition and high skew propeller. The influence of the Smagorinsky constant is also studied, which shows the results have a low sensitivity to it. Published by AIP Publishing. [http://dx.doi.org/10.1063/1.4974522]
\end{abstract}

\section{INTRODUCTION}

The numerical simulation methods for the marine propeller flow can be classified into two categories: the potential method and grid-based CFD (computational fluid mechanics) method. Among potential methods, the boundary element method (BEM) is the most accurate and popular one. ${ }^{1-3}$ Computational elements are placed only on boundaries, and the simulation is quite fast. The accuracy relies on the correct prediction of the wake surface, which represents the vorticity shedding from the blades. BEM faces difficulty for high loading conditions, where the viscous phenomenon is not negligible. It is also intricate to calculate the interaction effect between structures with BEM. ${ }^{4}$

The grid-based CFD methods for the marine propeller flow include the RANS (Reynolds Averaged Navier-Stokes) solver ${ }^{5,6}$ and DES (Detached Eddy Simulation) solver. ${ }^{7}$ They are more accurate than BEM in terms of the forces acting on the propeller. However, such methods depend on the generation of high-quality 3D mesh, which calls for experience and much time. Besides, the grid-based methods suffer numerical diffusion, and thus always underestimate the vorticity strength in the vortex flow. This can be overcome to some extent by increasing grid density, but at the expense of much more computational effort.

The vortex particle method (VPM) is a mesh free computational fluid dynamic method. Lagrangian vortex particles associated with vector strengths are used to model the vorticity field and its development. With VPM, the viscous diffusion can be modelled with the particle strength exchange (PSE)

\footnotetext{
a) Author to whom correspondence should be addressed. Electronic mail: wyg216@yeah.net.
}

technique ${ }^{8}$ and the numerical diffusion is avoided. Besides, no delicate mesh is needed. VPM was introduced for 2D problems in $1931^{9}$ and extended to 3D around $1985 .{ }^{10,11}$ Since then, VPM has been utilized to investigate the behavior of vortex rings ${ }^{12-14}$ and simple wall-bounded flows. ${ }^{15-17}$ It is inherently a method to simulate the vorticity field accurately. Implementing VPM for engineering applications is attractive and helpful. Although most of the existing works are direct numerical simulations (DNSs), in the 1990s a pioneering investigation for the large-eddy simulation (LES) using VPM has been conducted. ${ }^{18}$ The sub-grid scale (SGS) model was applied to modify the viscosity. Several unbounded vortex flows were simulated with the developed method.

Inspired by the previous works, ${ }^{19,20}$ Wang et al. ${ }^{21}$ combined VPM with low-order boundary element method (BEM) for the simulation of hydrofoils. They used VPM to model the slipstream of the hydrofoils while BEM was used for the body surface. Details of how to combine these two methods were discussed and the influences of the involved computation parameters were studied. The present work is devoted to applying the similar hybrid method to the calculation of open water characteristics of marine propellers, which means the blade surfaces are modelled with BEM while the slipstream is modelled with VPM. The problem for the marine propeller is more complicated than for the hydrofoil. The starting vortices of different blades may fuse and the slipstream of a propeller may need far more particles than a hydrofoil.

In the current work, the VPM is based on the large-eddy simulation (LES) technique with the Smagorinsky-Lilly SGS model. The rotational periodic boundary condition is used to reduce the particle number and computational effort. A two substeps particle redistribution scheme is developed for the cylindrical sector domain with rotational periodic boundary. 
The fast multipole method is also implemented and adjusted for rotational periodic boundary conditions. Open water characteristics of three propellers with different skews are computed and compared with the results obtained with BEM and experiments. The current study is a preliminary work to compute the interaction between marine propellers and afterwards structures with the hybrid method. It also contributes to further engineering applications of VPM.

\section{VORTEX PARTICLE METHOD}

\section{A. Direct numerical simulation using vortex particle method}

For an incompressible fluid, the Lagrangian form of the Navier-Stokes equation is (external force is ignored)

$$
\frac{\mathrm{D} \boldsymbol{u}}{\mathrm{D} t}=-\frac{1}{\rho} \nabla P+v \Delta \boldsymbol{u},
$$

where $\boldsymbol{u}$ is the velocity, $v$ is the kinematic viscosity, $t$ is the time, $\rho$ is the density, and $P$ is the pressure. Defining vorticity $\omega$ as

$$
\omega=\nabla \times \boldsymbol{u},
$$

and taking the curl of the Navier-Stokes equation (1), we can obtain the vorticity equation for the incompressible, Newtonian fluid of uniform density as ${ }^{22}$

$$
\frac{\mathrm{D} \omega}{\mathrm{D} t}=(\nabla \boldsymbol{u}) \cdot \omega+v \Delta \omega .
$$

The VPM is always based on the vorticity equation (3), and in which the vorticity field is modelled with Lagrangian, vector-valued particles,

$$
\omega(x)=\sum_{p} \alpha_{p} \zeta_{\varepsilon}\left(x-x_{p}\right),
$$

where $\boldsymbol{x}_{p}$ and $\boldsymbol{\alpha}_{p}$ stand for the positions and vector strengths of particles, and $\zeta_{\varepsilon}$ denotes the mollification function which depends on the particle's core size $\varepsilon$. The vector strength $\boldsymbol{\alpha}_{p}$ is the total vorticity contained in a particle's volume,

$$
\alpha_{p}=\int_{V_{p}} \omega \mathrm{d} V,
$$

where $V_{p}$ is the volume associated with the particle. The mollification function has the ability to smooth the vorticity field and stabilize the simulation. It is defined by

$$
\zeta_{\varepsilon}(x)=\frac{1}{\varepsilon^{3}} \zeta\left(\frac{|x|}{\varepsilon}\right) .
$$

In the present work, the Gaussian mollification function is adopted, which is

$$
\zeta(\lambda)=\frac{1}{(2 \pi)^{1.5}} \exp \left(-\frac{\lambda^{2}}{2}\right) .
$$

With Equations (3) and (4), the formulas governing the VPM simulation are obtained as

$$
\begin{gathered}
\frac{\mathrm{d} \boldsymbol{x}_{\boldsymbol{p}}}{\mathrm{d} t}=\boldsymbol{u}\left(\boldsymbol{x}_{\boldsymbol{p}}\right), \\
\frac{\mathrm{d} \boldsymbol{\alpha}_{\boldsymbol{p}}}{\mathrm{d} t}=\nabla \boldsymbol{u}\left(\boldsymbol{x}_{\boldsymbol{p}}\right) \cdot \boldsymbol{\alpha}_{p}+V_{p} v \Delta \omega\left(\boldsymbol{x}_{\boldsymbol{p}}\right) .
\end{gathered}
$$

The local velocity $\boldsymbol{u}$ in Equation (8) is obtained by summing up the free stream velocity and the induced velocity. The induced velocity is the summation of the contribution from every single vortex particle as follows:

$$
\boldsymbol{u}_{i}(\boldsymbol{x})=\sum_{p} \boldsymbol{K}_{\varepsilon}\left(\boldsymbol{x}-\boldsymbol{x}_{\boldsymbol{p}}\right) \times \boldsymbol{\alpha}_{\boldsymbol{p}},
$$

where the velocity kernel $\boldsymbol{K}_{\varepsilon}$ is obtained by applying the BiotSavart theory to the mollified vortex particle. Its form is

$$
\boldsymbol{K}_{\varepsilon}(\boldsymbol{x})=\frac{f\left(\frac{|x|}{\varepsilon}\right)}{|\boldsymbol{x}|^{3}} \boldsymbol{x} .
$$

With the adopted mollification function (7),

$f(\lambda)=\int_{0}^{\lambda} \zeta(s) s^{2} \mathrm{~d} s=\frac{1}{4 \pi}\left[\operatorname{erf}\left(\frac{\lambda}{\sqrt{2}}\right)-\sqrt{\frac{2}{\pi}} \lambda \exp \left(-\frac{\lambda^{2}}{2}\right)\right]$.

The gradient of the velocity is obtained with

$$
\nabla \boldsymbol{u}_{i}(\boldsymbol{x})=\sum_{p} \nabla \boldsymbol{K}_{\varepsilon}\left(\boldsymbol{x}-\boldsymbol{x}_{\boldsymbol{p}}\right) \times \boldsymbol{\alpha}_{\boldsymbol{p}}
$$

The particle strength exchange (PSE) technique ${ }^{8}$ is applied to handle the Laplacian operator $\Delta$ for the viscous diffusion in Equation (9), which states

$$
\Delta \omega(x) \approx \frac{2}{\varepsilon^{2}} \int \eta_{\varepsilon}(x-y)(\omega(y)-\omega(x)) \mathrm{d} y
$$

with $\eta_{\varepsilon}(\boldsymbol{x})=\varepsilon^{-3} \eta(|\boldsymbol{x}| / \varepsilon)$ and $\eta(\lambda)=-\lambda^{-1} \mathrm{~d} \zeta(\lambda) / \mathrm{d} \lambda$. Thus, we have

$$
V_{p} v \Delta \omega\left(\boldsymbol{x}_{\boldsymbol{p}}\right)=\frac{2 v}{\varepsilon^{2}} \sum_{q}\left(V_{p} \boldsymbol{\alpha}_{\boldsymbol{q}}-V_{q} \boldsymbol{\alpha}_{\boldsymbol{p}}\right) \eta_{\varepsilon}\left(\boldsymbol{x}_{\boldsymbol{p}}-\boldsymbol{x}_{\boldsymbol{q}}\right)
$$

With locations and strengths of the particles, the vorticity field can always be recovered as well as the velocity field according to Equations (4) and (10). The particles are updated according to Equations (8) and (9) for every time step as the simulation of the development of the vorticity field.

\section{B. Large-eddy simulation using the vortex particle method}

For the fluid with high Reynolds numbers, DNS needs a quite small grid scale or a large amount of particles. It is more practical to use LES for engineering applications. In LES, the field variable is split up into filtered and sub-filtered portions, which are denoted with a bar and a prime, respectively,

$$
\phi=\bar{\phi}+\phi^{\prime} \text {. }
$$

With an eddy-viscosity assumption, the LES filtered Navier-Stokes equation can be expressed as ${ }^{23}$

$$
\frac{\mathrm{D} \overline{\boldsymbol{u}}}{\mathrm{D} t}=-\frac{1}{\rho} \nabla \bar{P}+\left(v+v_{t}\right) \Delta \overline{\boldsymbol{u}},
$$

where $v_{t}$ is called SGS kinematic viscosity. With the Smagorinsky SGS model, ${ }^{24}$ it is

$$
v_{t}=\left(C_{S} \Delta\right)^{2} \sqrt{2 \bar{S}_{i j} \bar{S}_{i j}}
$$

where $\Delta$ is the grid scale, $C_{S}$ is the Smagorinsky constant, $\bar{S}_{i j}$ is the filtered strain rate tensor. As proposed by Winckelmans, ${ }^{18}$ 


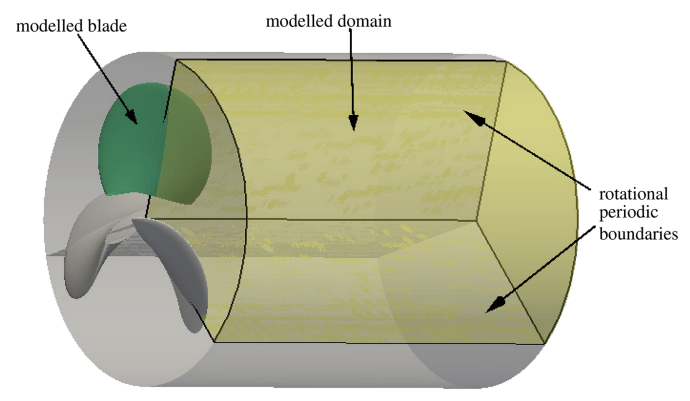

FIG. 1. The subdomain being modelled and the periodic boundary condition.

we use the particle's core size $\varepsilon$ for the grid scale $\Delta$. Different values have been proposed for $C_{S} \cdot{ }^{23-27} \mathrm{~A}$ value of $C_{S}=0.2$ is applied firstly and its influence is studied later in Section V. To maintain the conservativeness of Equation (15), when it is applied for a pair of particles, the root mean square of the two SGS viscosities is used.

Comparing Equation (17) with Equation (1), we found that only the total variable becomes the filtered variable and the kinematic viscosity is modified. The forms of the equations are the same. Thus, only by modifying the kinematic viscosity with the SGS model, we change the DNS-based VPM to the LES-based VPM.

\section{PARTICLE REDISTRIBUTION AND ROTATIONAL PERIODIC BOUNDARY CONDITION}

\section{A. Particle redistribution}

The overlapping condition, which states that the ratio of the core size $\varepsilon$ to the interparticle distance $h$ should be greater than unity, is the prerequisite for the convergence of VPM. ${ }^{11}$ The particle redistribution means the procedure in which a set of new particles located at regular positions is introduced to replace the old particles, for the purpose of maintaining the overlapping condition.

The vector strengths of the new particles are determined through a $3 \mathrm{D}$ interpolation,

$$
\boldsymbol{\alpha}_{p}=\sum_{q} \tilde{\boldsymbol{\alpha}}_{q} W\left(\frac{\boldsymbol{x}_{p}-\tilde{\boldsymbol{x}}_{q}}{h}\right),
$$

with the tilde meaning the quantities of the old particles. In the above formula, $W$ is the interpolation kernel. It can be built by a Cartesian tensor product of one-dimensional interpolation

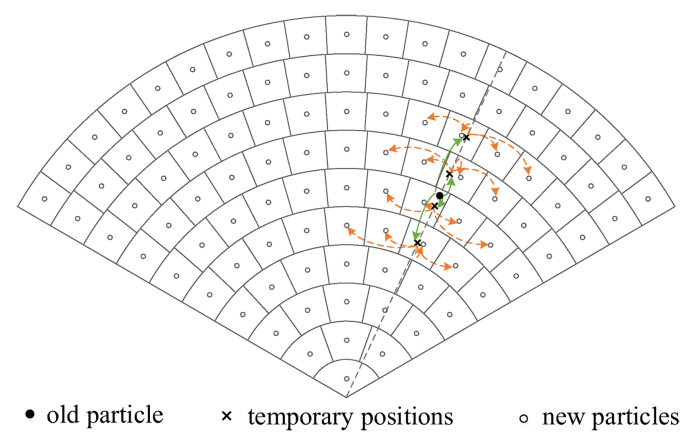

FIG. 2. The grid used in the particle redistribution and the redistribution procedure. functions,

$$
W(\boldsymbol{x})=W_{x}(x) \times W_{y}(y) \times W_{z}(z)
$$

where $x, y$, and $z$ are the components of $\boldsymbol{x}$, and $W_{x}, W_{y}$, and $W_{z}$ are one-dimensional interpolation functions along different directions.

The $M_{4}^{\prime}$ formula ${ }^{28}$ is always used for the one-dimensional interpolation function because of its ordinariness, smoothness, and high-order accuracy. It is

$$
M_{4}^{\prime}(x)= \begin{cases}0 & \text { if }|x|>2 \\ 0.5(2-|x|)^{2}(1-|x|) & \text { if } 1 \leq|x| \leq 2 . \\ 1-2.5 x^{2}+1.5|x|^{3} & \text { if }|x| \leq 1\end{cases}
$$

For more interpolation functions, please refer to Refs. 22 and 29.

\section{B. Rotational periodic boundary condition}

To calculate the open water characteristics of a marine propeller, only a subdomain corresponding to one blade is modelled. The rest are modelled with the rotational periodic boundary condition, as shown in Figure 1.

In the present work, the cross-section of the modelled subdomain is a sector. As particles go out from one periodic boundary, corresponding new ones come in from the other periodic boundary. When the induced velocity and velocity gradient are computed, the contribution from other subdomains is included by creating virtual particles according to the periodic condition.

\section{Particle redistribution with rotational periodic boundary condition}

Regular hexahedral cells are generated in the modelled subdomain, and new particles are placed at the center of the cells. The interparticle distance $h$ is used as the parameter to control the particle density during the particle redistribution. Uniform grids are used along axial and radial directions. Every radial strip is divided uniformly so that the cell's tangential length is around $h$. The grid and cells on the cross section are illustrated in Figure 2.

The mapped coordinates (or cylindrical coordinates) are used instead of the original Cartesian coordinates in the

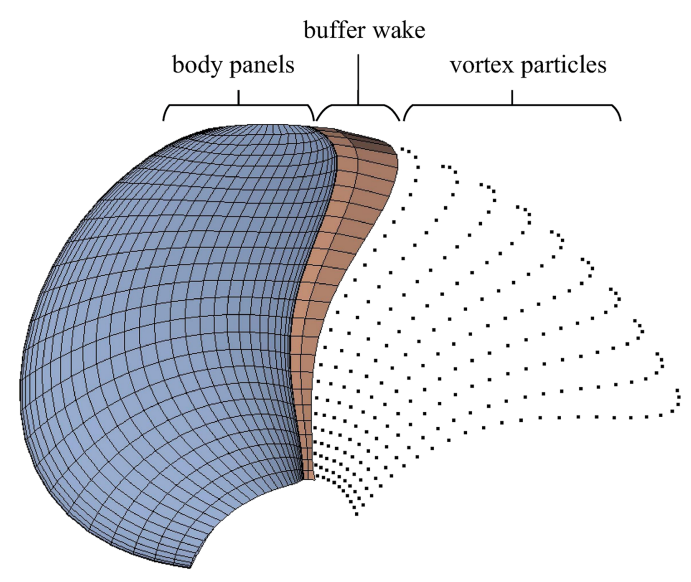

FIG. 3. Combination of VPM and BEM. 
TABLE I. Principal particulars of propellers.

\begin{tabular}{lccc}
\hline \hline Parameter & P4119 & KP505 & P4384 \\
\hline Diameter (model scale) (m) & 0.305 & 0.25 & 0.305 \\
Boss ratio & 0.20 & 0.18 & 0.20 \\
Pitch ratio at 0.7R & 1.084 & 0.997 & 1.200 \\
Number of blades & 3 & 5 & 5 \\
Angle of skew (deg) & 0 & 32 & 72 \\
Designed advance ratio & 0.833 & 0.7 & 0.889 \\
\hline \hline
\end{tabular}

interpolation. For the sake of simplicity, $x, r$, and $\theta$ are used to denote the axial, radial, and tangential coordinate, respectively. Instead of Equation (20), the interpolation kernel in Equation (19) is expressed as

$W\left(\frac{\boldsymbol{x}_{p}-\tilde{\boldsymbol{x}}_{q}}{h}\right)=W_{x}\left(\frac{x_{p}-\tilde{x}_{q}}{h}\right) \times W_{r}\left(\frac{r_{p}-\tilde{r}_{q}}{h}\right) \times W_{\theta}\left(\frac{\theta_{p}-\tilde{\theta}_{q}}{\mathrm{~d} \theta}\right)$,

while $W_{x}, W_{r}$, and $W_{\theta}$ all have the form of $M_{4}^{\prime}$ function.

The tangential interval $\mathrm{d} \theta$ is not identical for different radii. Thus, the tensor product cannot be applied directly. The $3 \mathrm{D}$ interpolation is conducted in two steps. First, the vector strength of an old particle is interpolated in the $x$ and $r$ directions to different $x$ and $r$ positions. Then, at every specific $x$ and $r$ position, one-dimensional interpolation is applied in the $\theta$ direction. This procedure is shown in Figure 2 .

Along the $r$ direction, a virtual symmetric condition is assumed at the position $r=0$. This means that the vortex strength is firstly interpolated to positions with $r<0$, and then the results are added to the positions with the radius equal to $|r|$.

The periodic boundary condition is also considered in the particle redistribution. Virtual cells (or particles) are placed out of the left periodic boundary. The strengths being interpolated to these cells are added to the corresponding particles near the right periodic boundary, and vice versa.

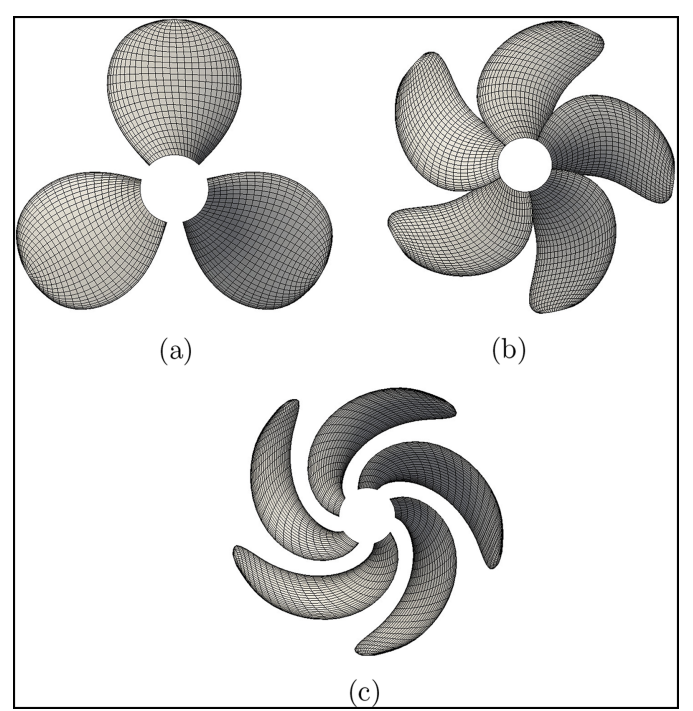

FIG. 4. Panel arrangement for propeller (a) P4119, (b) KP505, and (c) P4384.
TABLE II. Parameters for the simulations.

\begin{tabular}{lccc}
\hline \hline Parameter & P4119 & KP505 & P4384 \\
\hline Boundary layer thickness at 0.7R & $0.0096 \mathrm{D}$ & $0.0198 \mathrm{D}$ & $0.0075 \mathrm{D}$ \\
Particle core size & $0.01 \mathrm{D}$ & $0.02 \mathrm{D}$ & $0.008 \mathrm{D}$ \\
Number of particles from one panel & 15 & 9 & 24 \\
Redistribution interparticle distance & $0.009 \mathrm{D}$ & $0.015 \mathrm{D}$ & $0.008 \mathrm{D}$ \\
\hline \hline
\end{tabular}

\section{COMBINATION WITH BEM AND ACCELERATION ALGORITHM}

\section{A. Combination with BEM}

To avoid the complexity of the vortex particles near the solid boundary, the blade surface is modelled with BEM (boundary element method).

Boundary elements with constant dipole and source strength are placed on the blade surface. Panels with dipole strength are used to model the viscous wake shedding from the trailing edge. Dipole strengths on the wake panels are determined in accordance with the nonlinear Kutta condition. Afterwards, the wake panels are converted to vortex particles with the relationship between vorticity and dipole strength,

$$
\boldsymbol{\omega}=\boldsymbol{n} \times \nabla_{s} \mu,
$$

where $\boldsymbol{n}$ is the panel's normal, $\mu$ is the dipole strength, and $\nabla_{s}$ denotes the surface gradient on the panel. Such a hybrid method is sketched in Figure 3.

To consider the interaction between the boundary element system and vortex particle system, the following aspects are conducted during the simulation:

1. Including the particles' induced velocity when calculating the source strength on boundary elements.

2. Including the boundary elements' induced velocity and velocity gradient when convecting the vortex particles.

For more details of the combination of BEM and VPM, please refer to Refs. 19-21 and 30.

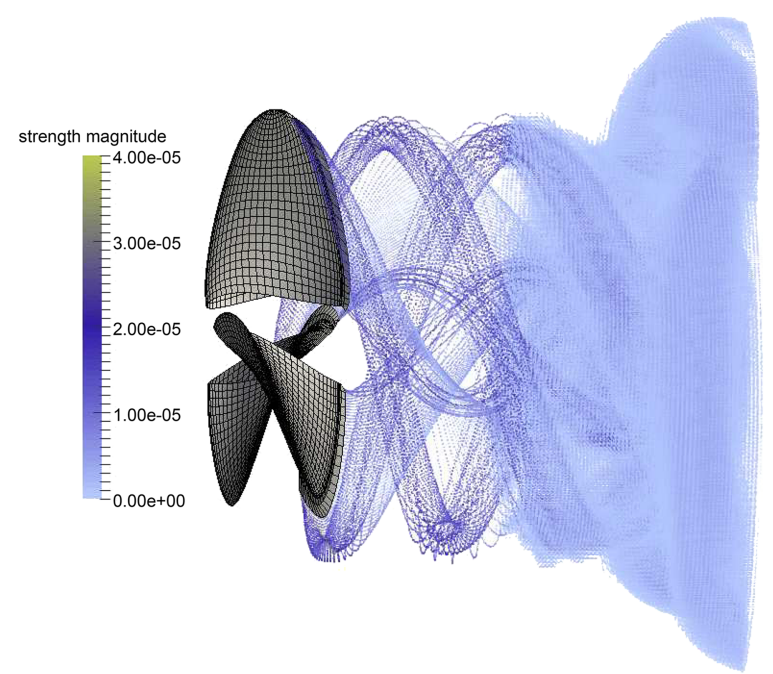

FIG. 5. Vortex particle distribution during the calculation for propeller P4119 at $J=0.5$. 


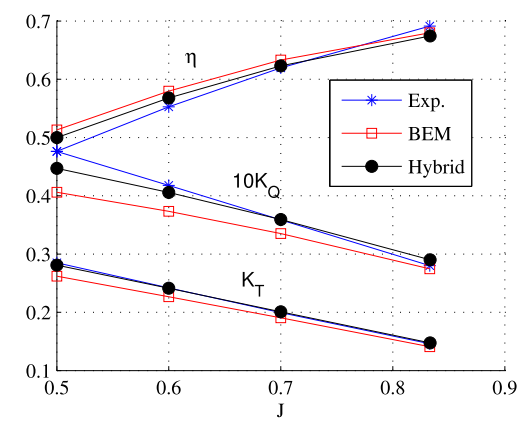

(a)

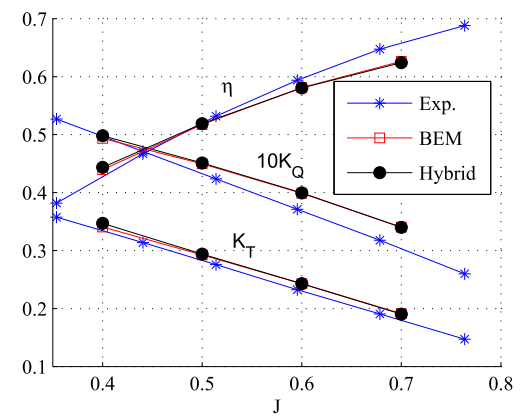

(b)

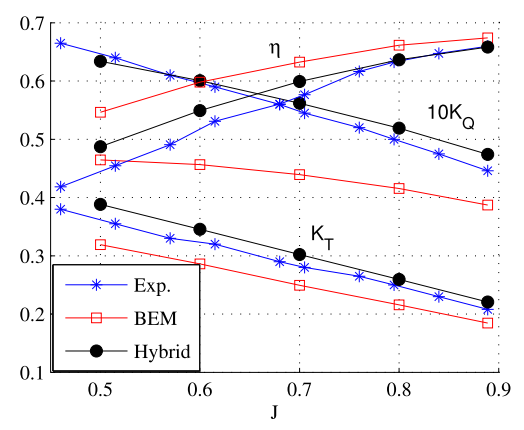

(c)
FIG. 6. Open water characteristics obtained with different methods for the propeller: (a) P4119, (b) KP505, and (c) P4384.

\section{B. Fast multipole method}

To accelerate the computation, we adopted the fast multipole method ${ }^{31,32}$ for the calculation of the induced velocity and velocity gradient of vortex particles. This method reduces the computational complexity from $O\left(N^{2}\right)$ to $O(N)$. The order of (19) is used for the expansions in the current work. The virtual vortex particles created outside of the modelled subdomain are included to compute the multipole expansions, but the local expansions are computed only for the box containing real particles.

\section{NUMERICAL RESULTS AND DISCUSSIONS}

With the current method, three marine propellers are simulated. They are P4119, ${ }^{33} \mathrm{KP} 505,{ }^{34}$ and P4384. ${ }^{35}$ Their principle particulars are listed in Table I. For the geometry discretization, 60 and 25 panels are used along the chordwise and radial directions, respectively. A cosine function is used to determine the chordwise panel distribution and a third order polynomial function is used for the radial direction. The forms

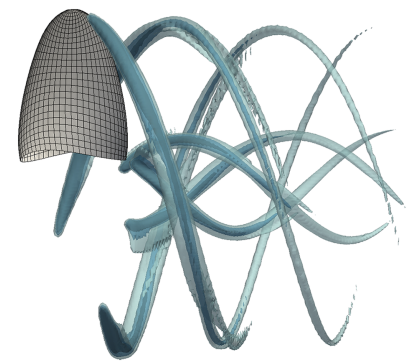

(a)

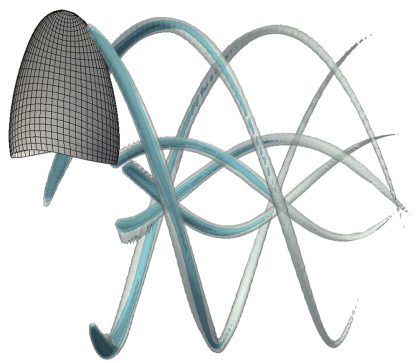

(b)
FIG. 7. Iso-surfaces of the vorticity strength after the propeller P4119 obtained with the hybrid method at (a) $J=0.7$ and (b) $J=0.833$. The value levels are $|\omega|=200,300$, and 400 . of the functions are

$$
\begin{aligned}
s_{i} & =\frac{c}{2}\left(1-\cos \left(\frac{2 \pi i}{N_{c}}\right)\right) \\
i & =0, \ldots, N_{c} / 2
\end{aligned}
$$

and

$$
\begin{aligned}
r_{j} & =f\left(\frac{j}{N_{R}}\right)\left(R-r_{h u b}\right)+r_{h u b} \\
f\left(\frac{j}{N_{R}}\right) & =-0.9563\left(\frac{j}{N_{R}}\right)^{3}+1.3790\left(\frac{j}{N_{R}}\right)^{2}+0.5773 \frac{j}{N_{R}} \\
j & =0, \ldots, N_{R},
\end{aligned}
$$

where $s_{i}$ is the chord coordinate of grid points on the section with the chord length $c, r_{j}$ is the radius of the $j$ th section, $f(\bullet)$ is the polynomial kernel function, $R$ and $r_{h u b}$ are the propeller and hub radius, respectively, and $N_{c}$ and $N_{R}$ are the chordwise and radial panel number, respectively. The cosine function concentrates on the panels at the leading and trailing edges. The coefficients in Equation (25) are determined to make the derivatives of the kernel distribution $f$ at the hub and tip equal to $\tan \left(30^{\circ}\right)$ and $\tan \left(25^{\circ}\right)$, respectively. Thus, the panels are concentrated at the hub and tip, and the change in panel size is smooth. Besides, an elliptical PDE is solved to improve the orthogonality of the

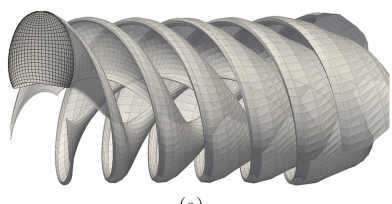

(a)

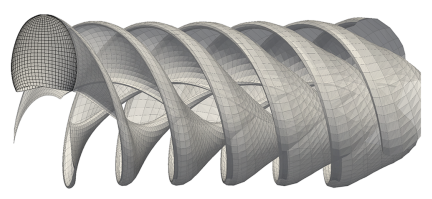

(b)
FIG. 8. Wake surfaces after the propeller P4119 obtained with BEM at (a) $J$ $=0.7$ and (b) $J=0.833$. 


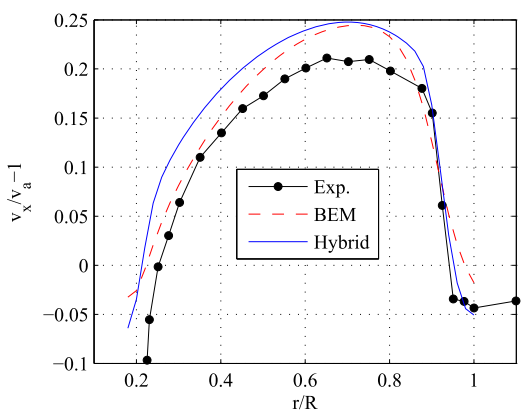

(a)

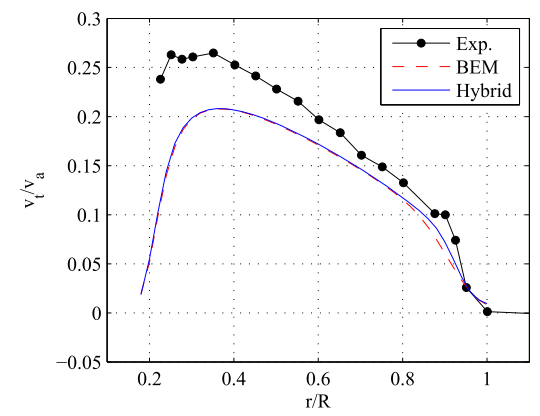

(b)
FIG. 9. Circumferential averaged nondimensional velocity after the propeller P4119 at $x / R=-0.3281$ and $J=0.7$ : (a) axial velocity and (b) tangential velocity. grid. The panel arrangements on the propellers are shown in Figure 4.

The time step is settled so that the propeller rotates $6^{\circ}$ every step. Such a time step size and the chosen panel numbers are enough to obtain the convergent results, according to previous work. ${ }^{36}$ Particles after $0.5 D$ ( $D$ stands for the propeller diameter) are redistributed every step with the interparticle distance $h$. Assuming that the shedding vorticity has a thickness comparable to the boundary layer, the particle core size is determined by the boundary layer thickness at radius position $r / R=0.7$. The boundary layer thickness is evaluated according to Schlichting ${ }^{37}$ as

$$
\delta=0.154 \operatorname{Re}^{-\frac{1}{7}} C,
$$

where $R e$ is the local Reynolds number and $C$ is the chord length.

The number of particles converted from one wake panel and the redistribution interparticle distance $h$ are set so that the particle spacing is smaller than the core size. The relative parameters for different propellers are listed in Table II. The particle distribution for propeller P4119 at $J=0.5$ is shown in Figure 5. The vortex region has a larger radius in the right of the figure. If we think the propeller advances in calm water, then the larger-radius region corresponds to the position where the propeller started suddenly from standstill. Thus, as shown in Figure 5, there are vortices on the left side of this region but no vortices on the right side. Because of the unbalanced radial velocity induced by the tip vortices on the left side, the fluid and vortices in this region move to the outer area and form the larger-radius region. The tip vortices and starting vortices of the blades interact with each other in this region and the vortex fusion happens. This always makes the
DNS-based VPM simulation crash with the current particle density and makes the LES-based VPM more expedient. In the simulation, the particles which are far enough after the propeller (one diameter in the present work) are removed. Therefore, the larger-radius region will be removed as the simulation runs further.

In the present work, the thrust coefficient $K_{T}$, torque coefficient $K_{Q}$, and the efficiency $\eta$ are used to represent the open water characteristics of the propeller. Their definitions are as follows:

$$
\begin{gathered}
K_{T}=\frac{T}{\rho n^{2} D^{4}} \\
K_{Q}=\frac{Q}{\rho n^{2} D^{5}} \\
\eta=\frac{T v_{a}}{Q \omega}=\frac{J K_{T}}{2 \pi K_{Q}}
\end{gathered}
$$

where $T$ and $Q$ are the thrust and torque generated by the propeller, respectively; $\rho$ is the fluid density, $n$ is the rotation rate with the unit of revolution per second (RPS), $D$ is the propeller's diameter, $v_{a}$ is the propeller's advance velocity, $\omega=2 \pi n$ is the angular velocity, and $J=\frac{v_{a}}{n D}$ is the advance ratio. The computation is considered to converge when the relative errors of $K_{T}$ and $K_{Q}$ of the last 10 steps with respect to the final step are all less than $0.1 \%$.

The open water characteristics obtained with the current method are given in Figure 6 with the results from BEM and experiments. With the same grid distribution on the blade surface, modelling the slipstream with VPM improves the results for all three propellers, especially for the high loading condition and high skew propeller. The vortex structures after the

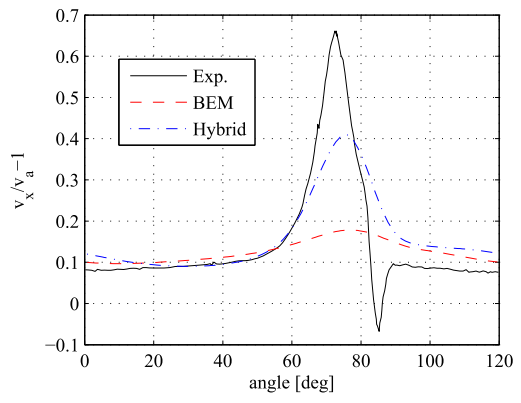

(a)

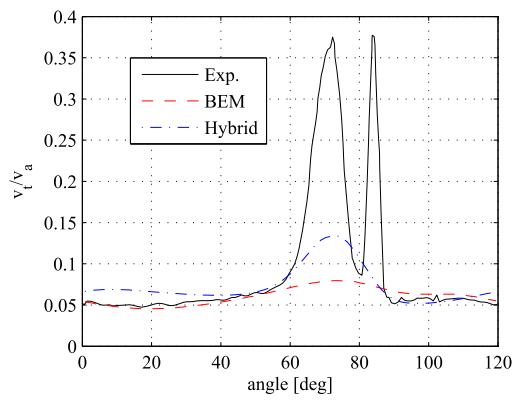

(b)
FIG. 10. Variation of velocities with phase angle for propeller P4119 at $J=0.833$ on the transverse plane at $x / R$ $=-0.3281$ : (a) axial velocity and (b) tangential velocity. 


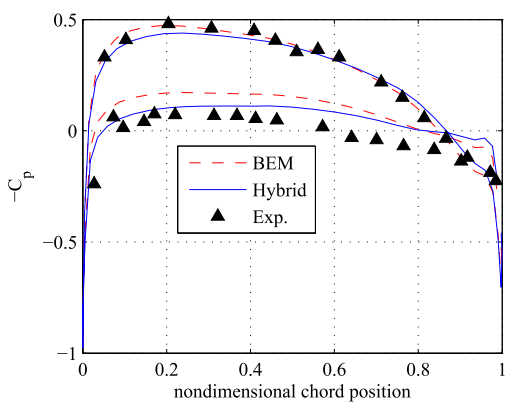

(a)

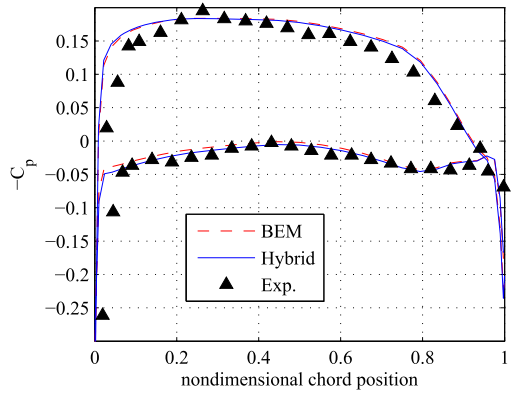

(b)

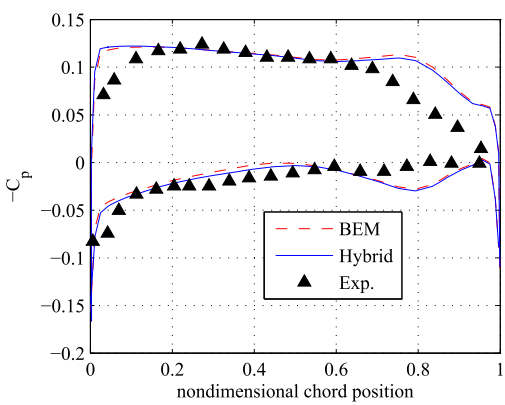

(c)

propeller P4119 obtained with the hybrid method are shown in Figure 7 through iso-surfaces of the vorticity strength. As a comparison, the wake surfaces obtained with BEM are shown in Figure 8. Due to the viscous diffusion and sub-grid dispersion, in the present method, the vorticity strength in the vortices decreases gradually as they are leaving the blade. On the other hand, in BEM the viscosity is ignored and the vortex strengths on the wake surface (represented by the circulation attached to the panel edges) stay unchangeable. Besides, in the hybrid method, the vortices thickness is modelled with the distribution of vorticity on particles and the mollification function. While in BEM, the thickness is hard to model because the vortices are represented by zero-thick helical filaments.

The obtained circumferential averaged non-dimensional axial and tangential velocities after the propeller P4119 are shown in Figure 9 together with the experimental data. For the denotations, $R$ represents the propeller radius, $x$-axis coincides with the rotational axis and directs forward, and $x=0$ corresponds to the propeller disk position. The flows after the propeller disk are accelerated. Both BEM and the hybrid method overestimate the axial velocity and underestimate the tangential velocity. The hybrid method leads to a better result near the tip vortex (around $0.9 R$ ). The detailed variation of the axial and tangential velocities with the phase angle at $0.9 R$ is given in Figure 10. The tip vortex is around the phase angle equalling to $70^{\circ}$. Around the tip vortex, the results obtained with the hybrid method correlate much better with the experimental data than the results from BEM. Thus, the hybrid method would lead to a more accurate tip vortex position, which is crucial for the prediction of open water characteristics. The pressure distributions on the propeller blade at different radial positions are shown in Figure 11, where the pressure coefficient is defined as

$$
C_{p}=\frac{p}{0.5 \rho\left(v_{a}^{2}+(\omega r)^{2}\right)}
$$

in which $p$ is the pressure and $r$ is the local radius. While the difference between the pressures obtained with BEM and the hybrid method is small, the ones from the hybrid method correlate a little better with the experimental results.

The computational time for two cases with the hybrid method and BEM is listed in Table III. For BEM, two revolutions of the wake are modelled. The wake alignment method is specifically for the steady calculation, e.g., the displacement accumulation method described by Wang et al. ${ }^{36}$ which leads to a convergence within 20 steps. All the computations ran on a computer with the Intel Xeon W3550

TABLE III. The computational time and simulation steps of the hybrid method (HM) and BEM.

\begin{tabular}{lcccc}
\hline \hline case & HM time (h) & HM steps & BEM time (s) & BEM steps \\
\hline P4119 at $J=0.7$ & 18.8 & 110 & 93 & 9 \\
KP505 at $J=0.6$ & 3.53 & 118 & 120 & 11 \\
\hline \hline
\end{tabular}

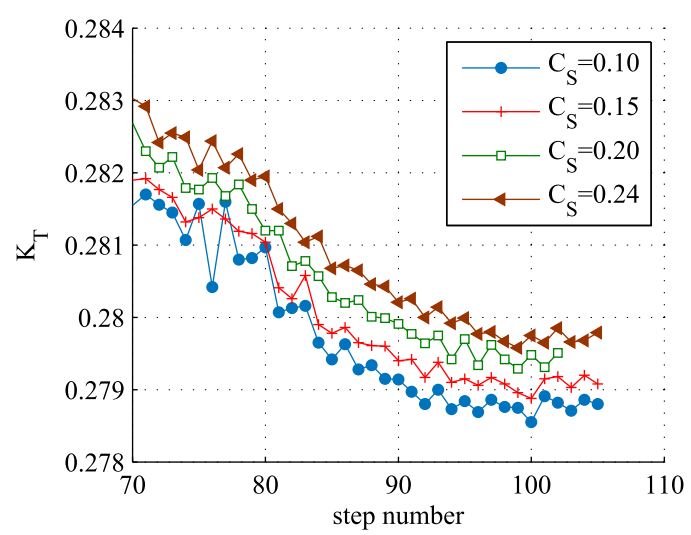

FIG. 12. The variation of thrust coefficient $K_{T}$ with different Smagorinsky constants $C_{S}$. 


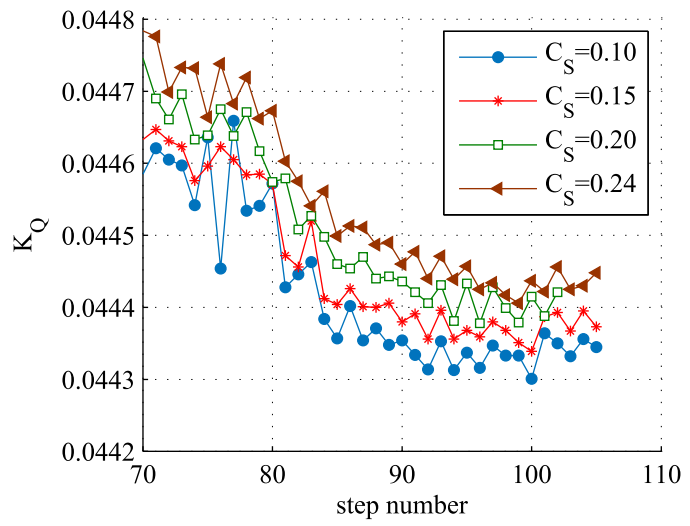

FIG. 13. The variation of torque coefficient $K_{Q}$ with different Smagorinsky constants $C_{S}$

octa-core processor. The hybrid method costs much more steps and computational time than BEM. However, the hybrid method calculates the steady case in an unsteady manner while BEM does it in a faster way. Thus, for the unsteady cases, the difference is expected to be smaller. And further technique to reduce the computational time of the hybrid method is under investigation.

As stated by Winckelmans, ${ }^{18}$ the Smagorinsky constant $C_{S}$ in Equation (18) lies in the range 0.1-0.24. In the present work, the value 0.2 is used for the previous calculation. Three more calculations for propeller P4119 at $J=0.5$ are carried out with $C_{S}=0.1,0.15$, and 0.24 , respectively. The variations of $K_{T}$ and $K_{Q}$ before convergence obtained with different $C_{S}$ values are shown in Figures 12 and 13, respectively. The $K_{T}$ and $K_{Q}$ both increase with constant. However, within the range $0.1-0.24$, the difference is less than $0.5 \%$, which is negligible.

\section{CONCLUSIONS}

A combination method of BEM and VPM is applied to the calculation of marine propellers open water characteristics. The VPM is based on the LES technique. The SmagorinskyLilly SGS model is adopted for the eddy viscosity. The rotational periodic boundary condition is applied, and only one blade and the corresponding cylindrical sector fluid domain are modelled. A two substeps particle redistribution scheme is developed to redistribute vortex particles on uniform positions in the cylindrical sector domain. The fast multipole method is also implemented, with creating virtual particles to handle the periodic boundary condition. The current method predicts more accurate open water characteristics of marine propellers when compared with BEM, especially for the high skew propeller and low advance ratios. The current work will be continued with the investigation of the slipstream flow detail and the simulation of the rotor-stator interaction using the presented method.

\section{ACKNOWLEDGMENTS}

The first author is sponsored by the China Scholarship Council.
${ }^{1}$ S. Berger, M. Druckenbrod, M. Pergande, and M. Abdel-Maksoud, "A twostage optimisation method for full-scale marine propellers working behind a ship," Ship Technol. Res. 61, 64-79 (2014).

${ }^{2}$ J. Baltazar, "On the modelling of the potential flow about wings and marine propellers using a boundary element method," Ph.D. thesis, Instituto Superior Técnico, Lisbon, Portugal, 2008.

${ }^{3}$ Y. Tian, "Leading edge vortex modeling and its effect on propulsor performance," Ph.D. thesis, UT, Austin, 2014.

${ }^{4} \mathrm{~L}$. He, "Numerical simulation of unsteady rotor/stator interaction and application to propeller/rudder combination," Ph.D. thesis, UT, Austin, 2010

${ }^{5}$ B.-w. Song, Y.-j. Wang, and W.-1. Tian, "Open water performance comparison between hub-type and hubless rim driven thrusters based on CFD method," Ocean Eng. 103, 55-63 (2015).

${ }^{6}$ M. Abdel-Maksoud and J. Heinke, "Scale effects on ducted propellers," in Proceedings of the 24th Symposium on Naval Hydrodynamics (National Academies Press, 2002), pp. 747-759.

${ }^{7}$ R. Muscari, A. Di Mascio, and R. Verzicco, "Modeling of vortex dynamics in the wake of a marine propeller," Comput. Fluids 73, 65-79 (2013).

${ }^{8}$ P. Degond and S. Mas-Gallic, "The weighted particle method for convectiondiffusion equations. I. The case of an isotropic viscosity," Math. Comput. 53, 485-507 (1989).

${ }^{9} \mathrm{~L}$. Rosenhead, "The formation of vortices from a surface of discontinuity," Proc. R. Soc. A 134, 170-192 (1931).

${ }^{10} \mathrm{C}$. Anderson and C. Greengard, "On vortex methods," SIAM J. Numer. Anal. 22, 413-440 (1985).

${ }^{11}$ J. T. Beale, "A convergent 3D vortex method with grid-free stretching," Math. Comput. 46, 401-424 (1986).

${ }^{12} \mathrm{G}$. S. Winckelmans, "Topics in vortex methods for the computation of three-and two-dimensional incompressible unsteady flows," Ph.D. thesis, California Institute of Technology, 1989.

${ }^{13}$ P. Chatelain, D. Kivotides, and A. Leonard, "Reconnection of colliding vortex rings," Phys. Rev. Lett. 90, 054501 (2003).

${ }^{14}$ M. Mammetti, R. Verzicco, and P. Orlandi, "The study of vortex ring/wall interaction for artificial nose improvement," in ESAIM: Proceedings (EDP Sciences, 1999), Vol. 7, pp. 258-269.

${ }^{15} \mathrm{P}$. Ploumhans and G. Winckelmans, "Vortex methods for high-resolution simulations of viscous flow past bluff bodies of general geometry," J. Comput. Phys. 165, 354-406 (2000).

${ }^{16}$ G.-H. Cottet, P. Koumoutsakos, and M. L. O. Salihi, "Vortex methods with spatially varying cores," J. Comput. Phys. 162, 164-185 (2000).

${ }^{17}$ P. Ploumhans, G. Winckelmans, J. K. Salmon, A. Leonard, and M. Warren, "Vortex methods for direct numerical simulation of three-dimensional bluff body flows: Application to the sphere at $\mathrm{Re}=300,500$, and 1000," $\mathrm{J}$. Comput. Phys. 178, 427-463 (2002).

${ }^{18} \mathrm{G}$. Winckelmans, "Some progress in large-eddy simulation using the 3D vortex particle method," CTR Annu. Res. Briefs 1995, 391-415 (1995).

${ }^{19}$ D. J. Willis, "An unsteady, accelerated, high order panel method with vortex particle wakes," Ph.D. thesis, Massachusetts Institute of Technology, 2006.

${ }^{20}$ J.-f. Tan and H.-w. Wang, "Simulating unsteady aerodynamics of helicopter rotor with panel/viscous vortex particle method," Aerosp. Sci. Technol. 30, 255-268 (2013).

${ }^{21}$ Y. Wang, M. Abdel-Maksoud, and B. Song, "A hybrid method combining boundary element and vortex particle methods," Comput. Fluids (submitted).

${ }^{22}$ G.-H. Cottet and P. D. Koumoutsakos, Vortex Methods: Theory and Practice (Cambridge University Press, 2000).

${ }^{23}$ Z. Yang, "Large-eddy simulation: Past, present and the future," Chin. J. Aeronaut. 28, 11-24 (2015)

${ }^{24}$ J. Smagorinsky, "General circulation experiments with the primitive equations. I. The basic experiment," Mon. Weather Rev. 91, 99-164 (1963).

${ }^{25}$ C.-H. Moeng and P. Sullivan, "Large eddy simulation," Encycl. Atmos. Sci. 1140, 1150 (2003).

${ }^{26} \mathrm{D}$. K. Lilly, "The representation of small scale turbulence in numerical simulation experiments," in Proceedings of the IBM Scientific Computing Symposium on Environmental Sciences (IBM, 1967), pp. 195-210.

${ }^{27}$ V. Canuto and Y. Cheng, "Determination of the Smagorinsky-Lilly constant CS," Phys. Fluids 9, 1368-1378 (1997).

${ }^{28}$ J. Monaghan, “Extrapolating B splines for interpolation," J. Comput. Phys. 60, 253-262 (1985).

${ }^{29} \mathrm{P}$. Koumoutsakos, "Inviscid axisymmetrization of an elliptical vortex," J. Comput. Phys. 138, 821-857 (1997). 
${ }^{30}$ D. J. Willis, J. Peraire, and J. K. White, "A combined pfft-multipole tree code, unsteady panel method with vortex particle wakes," Int. J. Numer. Methods Fluids 53, 1399-1422 (2000).

${ }^{31} \mathrm{H}$. Cheng, L. Greengard, and V. Rokhlin, "A fast adaptive multipole algorithm in three dimensions," J. Comput. Phys. 155, 468-498 (1999).

${ }^{32}$ R. Yokota, T. K. Sheel, and S. Obi, "Calculation of isotropic turbulence using a pure Lagrangian vortex method," J. Comput. Phys. 226, 1589-1606 (2007).

${ }^{33} \mathrm{~S}$. Jessup, "An experimental investigation of viscous aspects of propeller blade flow," Ph.D. thesis, Catholic University, Washington DC, USA, 1989.
${ }^{34}$ MOERI, Moeri container ship (KCS), 2008.

${ }^{35}$ R. J. Boswell, "Design, cavitation performance, and open-water performance of a series of research skewed propellers," Technical Report No.3339, NSRDC, Washington, DC, 1971.

${ }^{36}$ Y. Wang, M. Abdel-Maksoud, and B. Song, "Convergence of different wake alignment methods in a panel code for steady-state flows," J. Mar. Sci. Technol. 21, 567 (2016).

${ }^{37}$ H. Schlichting, Boundary-Layer Theory (McGraw-Hill, New York, USA, 1987). 